\title{
Be Positive: the Yoga Way
}

\author{
JPN Mishra* \\ Dean, School of Life Sciences, Central University of Gujarat, India
}

Submission: September 1, 2017; Published: September 08, 2017

*Corresponding author:JPN Mishra, Dean, School of Life Sciences, Central University of Gujarat, Gandhinagar-382030, Gujarat, India, Email: jpnmishra@cug.ac.in

\section{Opinion}

Our thinking have a profound effect on our physical and emotional health. People who constantly keep focusing, in their routine life, on negative thinking, lead themselves towards low self-esteem and depression, and they are prone to suffering with unrelenting stress. In the situation of negative thoughts and stress the phenomena of flight-or-flight is operating with full swing which creates imbalance in hormonal profile dominated by Adrenaline and Noradrenaline hormones, causing hyper physiological states associated with uncontrolled psychological distortions. Positive or negative thinking are processes not inherited. In fact it depends upon how we perceive any situation. It's a learned response, a kind of habit we develop over years of absorbing the attitudes of various kind of stimuli around us. Positive thinking helps keeping the physiological functions in the standard range and appropriate mind-body communication, which ultimately leads to the state of good psycho-physiological health. Contrary to that negative thinking causes detorioration in physiological functions as well as break down in the in the pathway of mind-body communication thereby causing various states of psychosomatic disorders. If we intend to achieve the target of happy and healthy living, we should strive to shed negative thinking and remain positive in our thinking and action too.

Major detrimental factors for negative thinking are exaggerating the circumstances, ignoring the positive aspects of the situation, personalizing every issue, either or thinking habit, over generalizing the people around us and premature jumping to conclusions. If we want to address these problems and factors it may involve major three steps- (i) Awareness, (II) Answering every negative thought without any delay or postponement, (III) Appropriate action. Six basic rules are involved in these three steps: (i) To place ourselves in a receptive state of mind, (II) To enlist the strong affirmations, (III) To always live in the present, (iv) To avoid negative phrases, (v) To remain firm on the positive opinion without least doubt, (vi) To remain accommodative. To achieve the above-mentioned target Yoga is a cost effective and easy to learn remedy and have its firm efficacy beyond doubt. Eight steps of Maharshi Patanjali's Yoga technique are there having the treasure of benefit to create a personality with positive thinking, which may be summarized as under:

1. Yamas \& Niyamas (code of conduct): helps the individual to get rid of any kind of fear and/or anxiety or stress.

2. Asanas: Keep the tone of neuromuscular system in dynamic state and modulates the organic functions which helps bringing in the state of homeostasis.

3. Pranayam: fulfills the energy requirement of the individual and brings in the mind and body in very proximity for proper communication between them.

4. Prathyahaar: Helps restraining the special senses from any kind of deviationfrom positive to negative.

5. Dharna: Helps in sitting appropriate goal of action of positive thinking.

6. Dhyan (Meditation): Most effective and vibrant practice promoting mind-body communication.

7. Samadhi: Bringing in the state of tranquility.

All kinds of sufferings, both physical and mental, are having their origin in the negative thinking. Whatever we have in our mind, only that will be exhibited by the body. Hence mind body communication is the key factor of good health and wellbeing, and that can be achieved by positive thinking only, and Yoga is the only tool which bring in the balanced state of mind body communication.

same quantum of light is flowing through every human being. Once we realize Light in our hearts we are ready to take steps and quantum jumps towards higher evolution taking us beyond biological/ Darwinian evolution. This will create a paradigm shift from 'survival of the fittest to leave behind the rest' to 'arrival of the best to lead the rest'. This is also the essential message of VYOMA: Vedanta, Yoga, Meditation and Awakening for all. 